RAGE expression specifically in macrophages failed to effectively cloak microlesions, resulting in neutrophil swarming.

Importantly, the cloaking response by macrophages resident in abdominal muscle was shown to protect from whole myofibre death following a minor myocyte injury. And in a mouse model for muscle dystrophy, caused by dystrophin deficiency, cloaking formations in the absence of neutrophils were detected around myocyte damage in diaphragms from 14-day-old mice. Depletion of tissue-resident macrophages led to premature onset of the disease, marked by extensive necrotic areas and neutrophil infiltrates in macrophage-depleted mice but not in control animals.

So, the authors suggest that tissues subject to ongoing mechanical stress and thus incidental cell damage rely on tissue protection from resident macrophages.

Lucy Bird

ORIGINAL ARTICLE Uderhardt, S. et al. Residen macrophages cloak tissue microlesions to prevent neutrophil-driven inflammatory damage. Cell. 177, 541-555 (2019)

using this reduced model indicated that TLR5 can deliver co-stimulatory signals to $T$ cells by activating AP1, $\mathrm{NF}-\mathrm{KB}$ and CREB transcription factors, leading the authors to predict that TLR5 may synergize with the TCR to induce productive activation of naive CD4 ${ }^{+} \mathrm{T}$ cells.

They confirmed these predictions in experiments in which naive human $\mathrm{CD}^{+}{ }^{+} \mathrm{T}$ cells were stimulated via the TCR alone or in combination with TLR5 or CD28 stimulation. Notably, combined TCR and TLR5 stimulation induced a similar activation response in naive $T$ cells to TCR and CD28 co-stimulation.

The authors propose that co-stimulation of T cells via TLR5 could serve as a rescue pathway to enable T cell activation in immunodeficient settings. Moreover, their findings help to explain why the TLR5 ligand flagellin has been shown to be a good adjuvant in vaccination models.

Yvonne Bordon

ORIGINAL ARTICLE Rodríguez-Jorge, O. et al. Cooperation between $T$ cell receptor and Tolllike receptor 5 signaling for $\mathrm{CD}^{+} \mathrm{T}$ cell activation. Sci. Signal. 12, eaar3641 (2019)

\title{
ILC3s take control in small intestine
}

Intestinal homeostasis requires the anti-inflammatory effects of regulatory $T\left(T_{\text {reg }}\right)$ cells, which are maintained by IL-2. Contrary to conventional wisdom regarding the role of $\mathrm{T}$ cells as the main producers of IL-2 in the intestines, Zhou et al. report that, in the small intestine, group 3 innate lymphoid cells (ILC3s) have a crucial role in IL-2 production in response to the microbiota.

Adult mice given IL-2-neutralizing antibodies for a two-week period had decreased numbers of $\mathrm{T}_{\text {reg }}$ cells and increased proliferation of $\mathrm{CD}^{+}$ $T$ cells throughout the gastrointestinal tract, together with increased production of IFN $\gamma$ and IL-17A. By contrast, mice with a lineage-specific deletion of IL-2 in T cells had similar effects in the large intestine only, which indicates the existence of another cellular source of IL-2 in the small intestine.

The major population of $\mathrm{IL}^{-} 2^{+}$cells in the small intestine had a phenotype consistent with ILC3s as assessed by flow cytometry. Furthermore, RNA sequencing and quantitative PCR of cell populations sorted from healthy mouse small intestine confirmed higher levels of $1 / 2$ expression in ILC3s than in T cells, B cells and dendritic cells. Mice with a specific deletion of IL-2 in ILC3s had decreased numbers of $\mathrm{T}_{\text {reg }}$ cells in the small intestine and increased numbers of effector CD4 ${ }^{+} \mathrm{T}$ cells.

To investigate how IL-2 production by ILC $3 \mathrm{~s}$ is regulated in the small intestine, the authors looked at specific pathogen-free (SPF) mice, SPF mice exposed to broad-spectrum antibiotics and germ-free mice. The latter two groups had

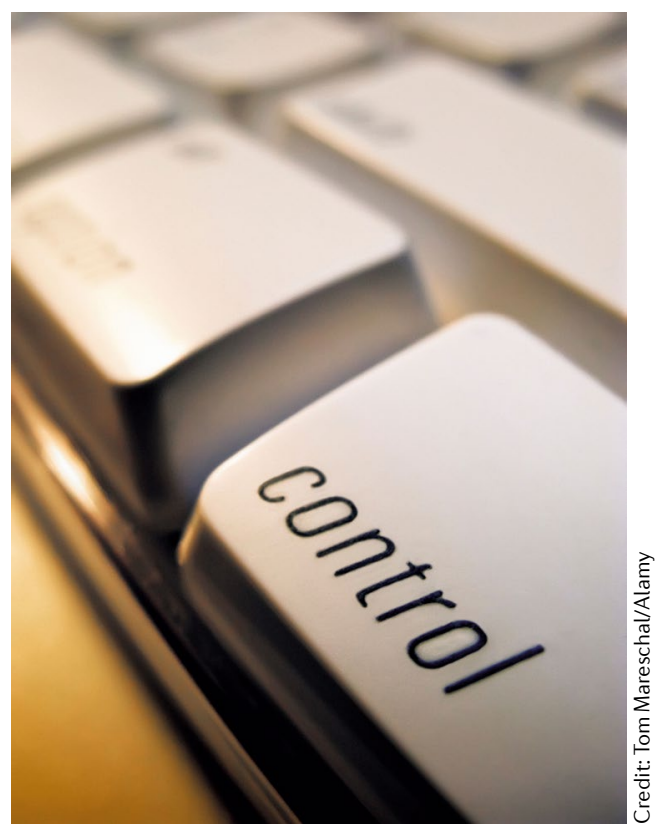

decreased IL-2 production by ILC3s, which suggests a role for the intestinal microbiota in inducing IL-2. Purified ILC3s from the small intestine could be induced to produce IL-2 at mRNA and protein levels by stimulation with $\mathrm{IL}-1 \beta$, and IL-1 $\beta$ was shown to be produced by macrophages in the small intestine through MYD88-dependent and NOD2-dependent pathways. Therefore, the data suggest that macrophage sensing of the intestinal microbiota through MYD88 and NOD2 leads to IL-1 $\beta$ production, which stimulates ILC3s to produce IL-2.

In keeping with this microbiota-dependent pathway, macrophages from the small intestine of germ-free mice had decreased IL-1 $\beta$ expression that could be partially restored by monocolonization with segmented filamentous bacteria. Further evidence of the role of IL-1 in this pathway came from mice with selective deletion of IL-1 receptor in NKp46+ ILC3s, which had decreased IL-2 production by ILC3s and decreased numbers of $\mathrm{T}_{\text {reg }}$ cells in the small intestine. No changes in $\mathrm{T}_{\text {reg }}$ cells were observed in the large intestine of these mice, which indicates that ILC3s have a tissue-specific role.

In an oral tolerance model of ovalbumin (OVA) feeding, the induction of OVA-specific induced $\mathrm{T}_{\text {reg }}$ cells in the small intestine was reduced in mice with ILC3-specific loss of IL-2. This failure to induce oral tolerance resulted in these mice mounting a delayed-type hypersensitivity response to OVA challenge in the periphery.

Extending these results to the human intestine, the authors looked at intestinal biopsies from healthy controls and individuals with a form of inflammatory bowel disease (IBD). The small intestine of individuals with IBD had decreased frequencies of $\mathrm{T}_{\text {reg }}$ cells and ILC3s, and the frequencies of these two cell types were positively correlated within individual biopsies. Purified ILC3s from inflamed regions of the small intestine in individuals with IBD had decreased levels of IL2 mRNA compared with ILC3s from non-inflamed regions of the same intestine.

This study identifies a mechanism of direct communication between ILC3s and $\mathrm{T}_{\text {reg }}$ cells that is specific to the small intestine and depends on microbial sensing by macrophages. This pathway has a non-redundant role in maintaining oral tolerance and preventing intestinal inflammation.

Kirsty Minton 